\title{
Characterizing Dense Suspensions using Image Analysis: A Case Study from the Pharmaceutical Industry
}

\author{
Nazia N. Khawaja ${ }^{1}$ and David J. Goldfarb ${ }^{2}$ \\ 1. Merck Research Laboratories, Preformulation Sciences, Rahway, NJ \\ 2. Merck Manufacturing Division, Center for Materials Sciences \& Engineering, Rahway, NJ
}

During the development of Project $X$, a dense suspension containing a poorly soluble active pharmaceutical ingredient (API), it was observed upon scale up that the oral suspension turned into a thick paste rather than a free-flowing suspension. This was not only a critical problem for the manufacturing process, but also a potential problem for the clinical performance of the drug product. The existing method of monitoring the manufacturing process through Particle Size Distribution (PSD) measurement by laser diffraction lacked the ability to distinguish between these batches. Scanning Electron Microscopy (SEM) was successful in identifying API morphology differences between these batches which attributed to the paste formation. The pasting was a result of changes made to the processing conditions during scale up which caused Ostwald ripening to occur.

SEM measurements, however, were not practical for use at the manufacturing site. The technique was not quantitative for such a dense suspension and used a relatively small sample size. The SYSMEX Image Analyzer (FPIA 3000) was successful in quantitatively and qualitatively monitoring the morphological changes within the oral suspension (Figure 1). The particle aspect ratio was one of the key values monitored during the manufacturing of Project X. It was found that an Aspect Ratio (min. distance/max. distance) greater than 0.4 at the Lower $10 \%$ of the particle size distribution indicated plate-like particles while an aspect ratio less than 0.4 indicated the non-desirable presence of rods. The images which were produced by the system further confirmed the presence/ absence of rods. With the aid of the SYSMEX image analyzer, process engineers were able to reduce the residence time of the product in the milling step, thus reducing the propensity for Ostwald ripening. Additonally, API solubility was minimized throughout the process by minimizing both surfactant concentration and temperature changes. In doing so, the manufacturing process for Project $\mathrm{X}$ was optimized to limit the formation of rods and therefore preventing the formation of paste. 

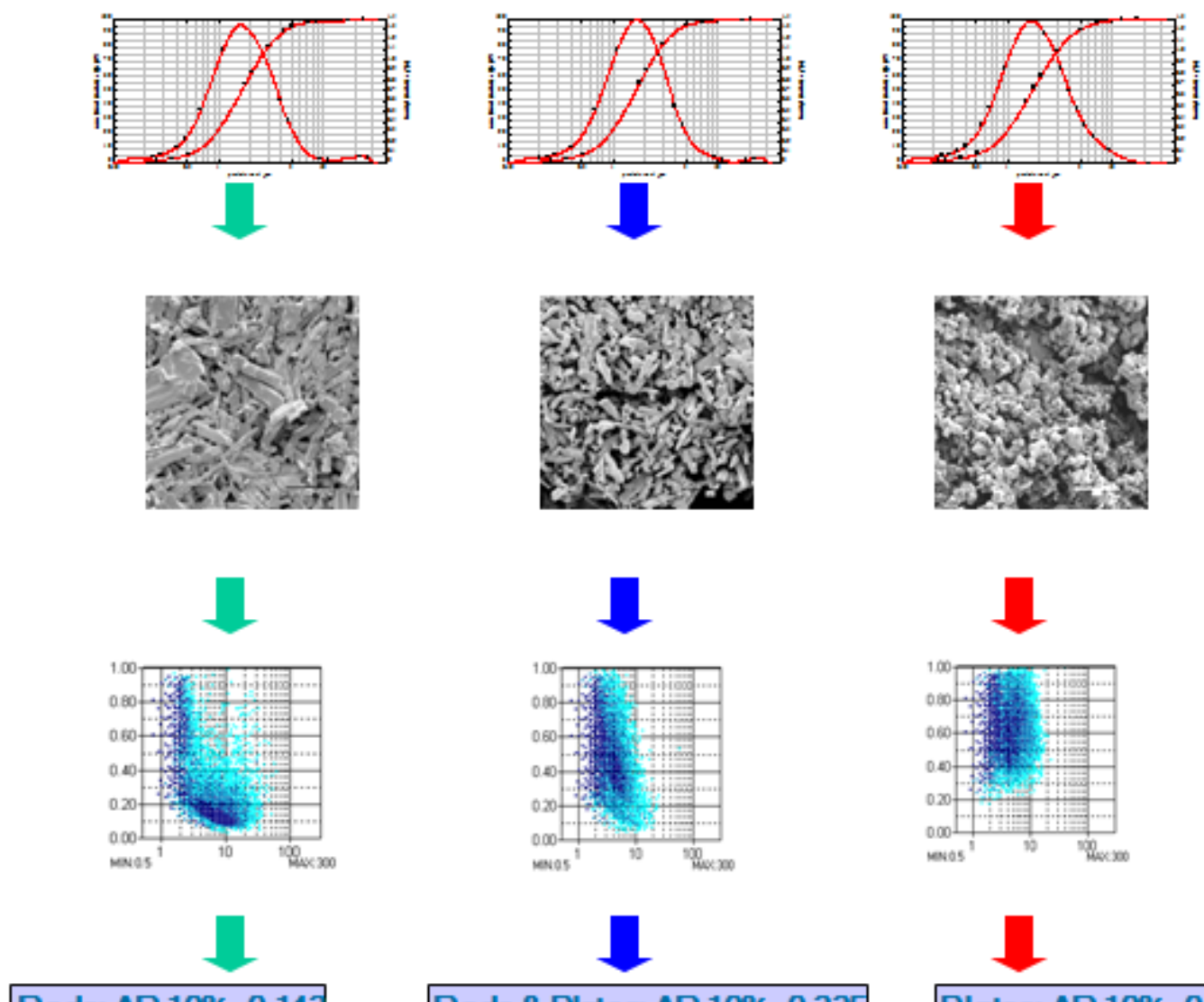

Rods \& Plates AR $10 \%=0.335$

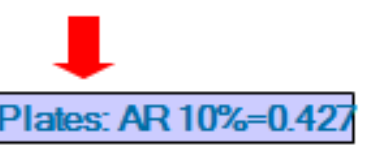

Figure 1. Three batches ( left to right) of decreasing viscosity are shown for Project X. PSD (top row) by laser diffraction is not able to distinguish between high viscosity and low viscosity samples. SEM images (middle row) qualitatively show that the high viscosity samples are due to the presence of rods in the formulation. The SYSMEX FPIA 3000 (bottom row) is able to quantify the rods in the formulation. 